\title{
Autistic adult outcomes on weight and body mass index: a large-scale online study
}

\author{
Felicity Sedgewick $^{1} \cdot$ Jenni Leppanen ${ }^{1} \cdot$ Kate Tchanturia $^{1,2,3}$
}

Received: 1 March 2019 / Accepted: 12 April 2019 / Published online: 7 May 2019

(c) The Author(s) 2019

\begin{abstract}
Purpose There has been a wealth of work on the weight outcomes of autistic children and young people, generally finding that they are more likely to be overweight or obese than their non-autistic counterparts. There has not been the same focussed study of the weight outcomes of autistic adults, however. This study, therefore, sought to examine the relationship between weight outcome and being autistic in adults.

Methods Data were collected as part of an online study looking at eating, autism, and relationships. 665 people gave demographic and mental health information, and group differences and robust regressions were conducted.

Results Autistic adults were more likely to be in non-healthy weight categories than their non-autistic counterparts, i.e., more likely to be underweight, overweight, or obese. There were no interactions between autism status and mental health impacting BMI, although both anxiety and depression predicted higher BMI in the sample overall.

Conclusions We conclude that while some weight patterns from childhood and adolescence continue into adulthood for autistic individuals, this is not necessarily a straightforward picture, and would benefit from further in-depth and qualitative study to understand the processes at play. The lack of interactions between mental health and autism, however, should provide professionals with confidence in supporting healthy weight management among autistic people.
\end{abstract}

Level of evidence Level III, cohort study.

Keywords Autism $\cdot$ Adult outcomes $\cdot$ Obesity $\cdot$ Eating $\cdot$ Weight $\cdot$ Mental health

\section{Introduction}

When discussing the weight outcomes of autistic people, there is a wealth of work showing that children and young people on the spectrum are more likely to be overweight or obese than their non-autistic peers [1,2]. There are a wide range of reasons for being overweight, but some of the most common factors-consuming more calories than you burn, low levels of physical activity-may particularly affect autistic people [3]. What has not previously been thoroughly explored, however, is whether these patterns extend

Kate Tchanturia

kate.tchanturia@kcl.ac.uk

1 King's College London, Institute of Psychiatry, Psychology and Neuroscience, Psychological Medicine, London, UK

2 South London and Maudsley NHS Trust EDU, London, UK

3 Psychology Department, Illia State University, Tbilisi, Georgia into adulthood, or their relationships with factors known to influence weight outcomes in non-autistic people.

Autistic children and young people often have very different diets to non-autistic children and young people, consuming a more limited range of foods [4], although this pattern is not universal [5]. Those foods which are often preferred by autistic children and young people tend to have a higher calorie count or have lower nutritional value [6]. Although it is common for children and young people to enjoy hobbies such as videogames, autistic children and young people engage in less physical activity than their non-autistic peers [7], tending to spend their time with their autistic and nonautistic friends in more sedentary activities [8].

To our knowledge, there are to date no studies which frame their work specifically as studying weight outcomes in autistic adults, but there is work which has included these data in exploring health outcomes generally. This work finds that autistic adults are more likely to be overweight/obese than nonautistic adults, along with also being more likely to have a wide range of health complaints $[9,10]$. Most adult outcome 
work has focussed on cognitive and social outcomes [11-13], but health outcome work generally shows that autistic adults have more difficulties. Work in non-autistic populations has shown that people with multiple health challenges are more likely to be in the overweight/obese categories [14, 15], and this is likely to also be the case for autistic adults.

Alongside physical health, mental health conditions have also been shown to be linked to higher BMI (body mass index) $[16,17]$ in non-autistic individuals. Autistic people are more likely to experience poor mental health than their non-autistic counterparts, with higher levels of anxiety $[18,19]$ and depression $[20,21]$ being particularly common. It is, therefore, possible that this interaction between mental health issues and increased BMI is also the case for autistic adults, but this has not been previously investigated.

One mental health condition which is by definition associated with low rather than high BMI is anorexia nervosa (AN), and connections between autism and AN have been suggested and explored since the 1980s [22]. This exploration has normally taken the form of conceptualising AN as a 'female form' of autism [23], with similarities in cognitive profiles-setshifting difficulties [24], detail-focussed processing [25, 26], and theory of mind challenges [27]—and in social difficulties $[28,29]$. These studies have, however, exclusively used female participants, as women are more likely to develop AN [30], compared to autism being more commonly diagnosed in males due to well-recognised diagnostic biases [31]. Furthermore, research looking at the overlap between the two conditions has very much focussed on the presence and level of autistic traits in AN populations, with some studies finding up to $23 \%$ of women receiving in-patient treatment for AN meet clinical criteria for autism $[32,33]$. This means that to date, there is no published research on how likely autistic people are to meet the criteria for AN of having a BMI below 18.5 [34].

This study, therefore, sought to examine all weight category outcomes of autistic and non-autistic adults, and the potential impact of mental health on these outcomes. Our hypotheses were:

1. Autistic people would on average have a higher BMI than non-autistic people, and would be more likely to be in the higher weight categories,

2. People with more mental health difficulties would have a higher BMI than those without mental health difficulties,

3. There would be an interaction between autism status and mental health resulting in higher BMI outcomes

\section{Method}

\section{Participants}

Six hundred and sixty-five people between the ages of 18 and 81 were included in the analysis, after the exclusion of 276 participants who self-reported having an eating disorder diagnosis. Of these $665,335(50.38 \%)$ reported that they were autistic, and $330(49.62 \%)$ reported no autism diagnosis. Gender, ethnicity, employment status, and other reported diagnoses among the two groups can be seen in Table 1.

Participants were recruited online through social media (Twitter, Facebook) and through online advertising on the

Table 1 Demographic characteristics of the sample by group (autistic, non-autistic)

\begin{tabular}{|c|c|c|}
\hline & Autistic $(n=335)$ & Non-autistic $(n=330)$ \\
\hline \multicolumn{3}{|l|}{ Age } \\
\hline Range & $18.12-71.42$ & $18.29-81.29$ \\
\hline $\mathrm{M}(\mathrm{SD})$ & $34.06(10.86)$ & $32.67(11.25)$ \\
\hline \multicolumn{3}{|l|}{ Gender } \\
\hline Male & $53(15.82 \%)$ & $53(16.06 \%)$ \\
\hline Female & $195(58.21 \%)$ & $266(80.61 \%)$ \\
\hline Non-binary & $86(25.67 \%)$ & $12(3.63 \%)$ \\
\hline \multicolumn{3}{|l|}{ Ethnicity } \\
\hline White & $246(73.43 \%)$ & $262(79.39 \%)$ \\
\hline Asian & $8(2.38 \%)$ & $24(7.27 \%)$ \\
\hline Black & $3(0.90 \%)$ & $3(0.91 \%)$ \\
\hline Latinx & $3(0.90 \%)$ & $1(0.30 \%)$ \\
\hline Mixed & $14(4.18 \%)$ & $9(2.73 \%)$ \\
\hline No answer & $59(17.61 \%)$ & $31(9.39 \%)$ \\
\hline \multicolumn{3}{|l|}{ Employment status } \\
\hline Full time & $89(26.57 \%)$ & $177(53.63 \%)$ \\
\hline Part time & $40(11.94 \%)$ & $27(8.18 \%)$ \\
\hline Student & $60(17.91 \%)$ & $91(27.57 \%)$ \\
\hline Self-employed & $39(11.64 \%)$ & $14(4.24 \%)$ \\
\hline Unemployed & $73(21.79 \%)$ & $12(3.63 \%)$ \\
\hline Retired & $8(2.38 \%)$ & $4(1.21 \%)$ \\
\hline Other & $23(6.87 \%)$ & $5(1.52 \%)$ \\
\hline \multicolumn{3}{|l|}{ Eating-related diagnoses } \\
\hline Diabetes & $21(6.27 \%)$ & $4(1.21 \%)$ \\
\hline Coeliac disease & $3(0.89 \%)$ & $1(0.30 \%)$ \\
\hline Crohn's disease & $2(0.60 \%)$ & $1(0.30 \%)$ \\
\hline Ehlers-Danlos syndrome & $2(0.60 \%)$ & $0(0.00 \%)$ \\
\hline $\begin{array}{l}\text { Food intolerances/aller- } \\
\text { gies }\end{array}$ & $104(31.04 \%)$ & $48(14.54 \%)$ \\
\hline Irritable bowel syndrome & $16(4.77 \%)$ & $8(2.42 \%)$ \\
\hline \multicolumn{3}{|l|}{ AQ score } \\
\hline Range & $4-28$ & $0-25$ \\
\hline $\mathrm{M}(\mathrm{SD})$ & $20.98(3.83)$ & $8.93(5.68)$ \\
\hline
\end{tabular}


King's College website and email circulars. Ethical approval was obtained from the King's Psychiatry, Nursing and Midwifery Research Ethics Committee (LRS-17/18-5292). All participants read a full information page before taking part in the study, and were informed that completing the study would be taken as consent for the use of their data. Participants also completed a written informed consent page before starting the survey. All procedures were conducted in accordance with the latest version of the Declaration of Helsinki.

\section{Measures}

\section{Demographics}

Participants completed a demographics questionnaire, including their age, height, weight, ethnicity, and employment status.

$A Q:$ The Autism Quotient-28 item version [35] is a 28-item self-report screening questionnaire assessing the presence and level of autism symptomatology an individual experiences. Answers are given on a Likert scale from 'Very accurate' to 'Very inaccurate' and are then scored 1 or 0 depending on the direction of the question. Higher scores reflect more autistic symptomatology.

\section{EDE-Q}

The Eating Disorder Examination Self-report Questionnaire [36] is a 36-item self-report questionnaire assessing eating disorder psychopathology over the past 28 days. Participants score the frequency of their behaviours or thoughts from '0' days' to 'Every day'. Higher scores reflect greater eating disorder symptomatology.

\section{HADS}

The Hospital Anxiety and Depression Scale [37] is a 14-item self-report questionnaire assessing levels of both anxiety and depression over the past 2 weeks. Answers are scored from 0 (not anxious/depressed) to 3 (very anxious/depressed) on each item for a maximum score of 42 . Higher scores reflect higher anxiety and depression levels.

\section{General procedure}

Participants all completed the study online, at their own pace and in a place of their preference. The data were collected as part of a larger study. Participants completed demographic information, the AQ, the EDE-Q, and the HADS online.

\section{Data analysis}

All data analyses were conducted with $\mathrm{R}$ [38]. Group differences in demographic and clinical characteristics were explored with $t$ tests. Weight category outcomes according to autism status were investigated using ordinal logistic regression [39]. Impact of self-reported mental health and autism status on BMI outcomes was investigated using robust regression [40]. Separate robust regression models were built to examine the impact of self-reported anxiety (HADS anxiety), depression (HADS depression), and eating disorder symptomatology (EDEQ total) along with autism status on BMI. Separate Spearman's correlation analyses were conducted to examine associations between AQ-28 scores and BMI within the two groups. Significance level was set at $p<0.05$.

\section{Results}

\section{Demographics}

Participants were matched on age, $t(664)=-1.62, p=0.11$. They were not matched on AQ score, with those who reported being autistic scoring significantly higher than those who reported being non-autistic, $t(664)=-31.22$, $p<0.001$.

\section{Weight outcomes}

The numbers of autistic and non-autistic people in each weight category can be seen in Table 2. Autistic people had a higher average BMI than non-autistic people, $t(664)=-4.03, p<0.001$. Within the autistic group, higher AQ scores were correlated with lower BMI, $r=-2.51$,

Table 2 BMI and weight category outcomes by group (autistic, nonautistic)

\begin{tabular}{lll}
\hline & Autistic $(n=335)$ & Non-autistic $(n=330)$ \\
\hline BMI & & \\
Range & $14.56-72.31$ & $15.06-63.82$ \\
M (SD) & $28.01(8.36)^{* * *}$ & $25.66(6.49)$ \\
Weight category & & \\
Underweight & $18(5.37 \%)^{* * *}$ & $9(2.73 \%)$ \\
Healthy weight & $126(37.61 \%)$ & $189(57.28 \%)$ \\
Overweight & $86(25.67 \%)^{* * *}$ & $71(21.52 \%)$ \\
Obese & $104(31.04 \%)^{* * *}$ & $61(18.48 \%)$ \\
\hline *Denotes significance at the 0.05 level & \\
$* *$ Denotes significance at the 0.01 level & \\
$* * *$ Denotes significance at the 0.001 level &
\end{tabular}


$p=0.01$. Within the non-autistic group, there was no correlation between AQ score and BMI, $r=1.43, p=0.15$.

A categorical outcome regression showed that being autistic had a significant impact on likely weight category outcome. Taking healthy weight as the baseline, autistic people were more likely to be either underweight $(t=-14.20$, $p<0.001$ ), overweight $(t=3.26, p<0.001)$, or in obesity range $(t=11.72, p<0.001)$ than non-autistic people. Being autistic was linked to a $58.84 \%$ greater chance of being in the overweight/obese weight categories than healthy weight category.

\section{Mental health and BMI}

Scores on mental health and clinical measures can be seen in Table 3. Autistic people scored more highly than non-autistic people on both the anxiety, $t(664)=-12.35, p<0.001$, and depression subscales of the HADS, $t(664)=-10.85$, $p<0.001$. There were no significant differences between the groups on the EDE-Q, $t(664)=-1.14, p=0.25$.

There was a significant main effect of autism status controlling for anxiety on BMI, $F(1)=9.70, p=0.002$, but no main effect of anxiety score, $F(1)=1.77, p=0.18$, and no interaction between autism status and anxiety score, $F(1)=0.83, p=0.36$.

There was significant main effect of autism status on BMI controlling for depression, $F(1)=5.800, p=0.2$, and also a significant main effect of depression score on BMI, $F(1)=13.17, p<0.001$, but no significant interaction between autism status and depression score, $F(1)=0.02$, $p=0.90$.

There was a significant main effect of autism status controlling for EDE-Q, $F(1)=16.15, p<0.001$, and also a main effect of EDE-Q score on BMI, $F(1)=129.46, p<0.001$, but

Table 3 Mental health and clinical scores by group (autistic, nonautistic)

\begin{tabular}{lll}
\hline & Autistic $(n=335)$ & Non-autistic $(n=330)$ \\
\hline $\begin{array}{ll}\text { HADS anxiety } \\
\text { Range }\end{array}$ & $0-21$ & $0-21$ \\
M (SD) & $11.81(4.45)^{* * *}$ & $7.55(4.44)$ \\
HADS depression & & \\
Range & $0-21$ & $0-18$ \\
M (SD) & $7.23(3.87)^{* * *}$ & $4.13(3.50)$ \\
EDEQ & & \\
Range & $0-5.68$ & $0-5.68$ \\
M (SD) & $1.77(1.39)$ & $1.66(1.21)$ \\
\hline
\end{tabular}

*Denotes significance at the 0.05 level

**Denotes significance at the 0.01 level

***Denotes significance at the 0.001 level no significant interaction between autism status and EDE-Q score, $F(1)=0.65, p=0.42$.

\section{Discussion}

The findings of this study suggest that autistic adults are less likely than non-autistic adults to have a healthy BMI, being more likely to be both under- and overweight. This finding draws together the results of two disparate fields of research - that on obesity in autism, and that on links between anorexia and autism-in ways which will be discussed in detail below. Higher BMI was linked to mental health difficulties in both autistic and non-autistic participants, but interestingly, there was no combined effect of being autistic and having mental health issues on BMI.

Our finding that autistic adults were found to be more likely to have BMI scores in the overweight or obese categories than non-autistic people is in line with earlier work which has shown that autistic children, adolescents, and adults are more likely to be overweight or obese than their non-autistic counterparts $[2,3,10]$. This is the first large-scale online study to look at adult weight outcomes in autistic people, and the results suggest that the weight patterns established in early life might continue into adulthood. In young autistic people, being overweight/obese is linked to lower activity levels [7], greater caloric intake [5], and preferences for low-nutrition foods [4]. Considering that difficulties with and a dislike of change is a well-recognised feature of autism [34], it may well be that these behavioural patterns are continued into adulthood, meaning that overweight/obese status is also maintained.

The seemingly contrary finding that autistic people are also more likely to be in the underweight category than the healthy weight category, however, can also be explained in terms of behaviour patterns being maintained from adolescence. There is a wealth of work examining links between anorexia nervosa, an illness characterised by low body weight and the pursuit of extreme thinness [34], and autism or autistic traits $[32,33,41]$. This research has shown that up to $23 \%$ of women with severe and enduring anorexia, i.e., those who maintain very low BMI for many years, have clinically significant levels of autism traits [32]. This association between autism and anorexia has been linked to similar cognitive profiles in the two conditions [27, 42-44]. It may, therefore, be that those autistic people, especially autistic women, who develop an eating disorder are more likely to fall into the lowest BMI category anyway and, therefore, find it hardest to change their behaviour pattern to achieve a healthy weight regardless of ongoing ED. There is also some evidence that children with developmental disabilities are more likely to be in the under- as well as overweight 
categories [45], a pattern which may also be maintained into adulthood.

This interpretation is supported by the finding that within the autistic group, higher AQ score was correlated with lower BMI, suggesting that more severely autistic people are more likely to have restricted calorie intake. Wider literature proposes a range of possible reasons for this, particularly around sensory sensitivities. That autistic people are often hyper- or hypo-sensitive to touch, taste, smell, and light is well-documented and was included as a diagnostic criterion in the new DSM-V [34]. These sensitivities may contribute to the restricted dietary range often seen in autistic children [4], and potentially result in the same in autistic adults, as people seek to avoid unpleasant or overstimulating foods. This in turn potentially leads to more acute malnutrition and low BMI amongst those with more sensory challenges associated with higher levels of autism symptomatology.

These sensory sensitivities may also play a role in the higher numbers of autistic adults who were in the overweight/obese categories as well as those in the underweight category. Just as some autistic people may be underweight due to sensory avoidance of unpleasant or overwhelming stimuli, some autistic people may be overweight/obese due to sensory seeking of foods which are pleasant to them. Considering work which has shown that, regardless of autism status, people find more emotional satisfaction from foods associated with their childhoods [46, 47] and that autistic children often have diets containing relatively high calorie foods [6], it is reasonable to assume that autistic adults who sensory seek through food tend to go for those same high calorie items, contributing to weight gain.

It was somewhat surprising that BMI outcome was not impacted by the interaction between autism and mental health. Individuals with mental health issues are often found to have higher BMI than those without mental health issues $[16,17]$, as are autistic people [10]. Considering that autistic people are more likely to have mental health issues than non-autistic people [18], a pattern which was also present in this study, it would be reasonable to predict that autistic people with mental health issues would have higher BMI scores than non-autistic people with mental health issues or autistic people without mental health issues. That this is not the case, however, is promising in that it suggests that there are not a set of unique processes occurring for autistic people with mental health issues regarding their weight outcomes, and therefore, weight interventions which already exist can be implemented with confidence. It is also the case that anxiety levels in our non-autistic sample were higher than the expected population norm, sitting at 7.55 in our sample compared to 4.4-5.0 in normative data [37]. This may be because the majority of participants were female, and women are known to experience higher levels of anxiety than men [48].
While this is generally a strong study due to the large sample size, there are some limitations to the research. First, the data come entirely from self-report, which raises the possibility of inaccurate answers, especially regarding weight. It is common for people to be unclear as to their weight in a way that it is not for their height, and therefore, it may be that there are some people who either underor over-reported their weight. Despite this, the broad range of BMI scores in both the autistic and non-autistic samples suggest that the overall sample is representative even if individual scores are somewhat off. Second, the groups were not matched on demographic variables such as gender or employment status. This is to be expected, however, considering work showing that autistic people are more likely to be gender non-conforming than nonautistic people [49-51] and that they can struggle to maintain full-time employment [52, 53], and therefore, these differences are representative of the population. Third, this study lacks any qualitative data, so we are unable to discuss what experiences and drivers people felt contributed to their weight in their own words. Future work exploring this topic would be valuable, as people cannot be supported in living healthier lives if we do not know what causes unhealthy behaviours in the first place. Fourth, this work captures people's weights at just one point in time, rather than being longitudinal, and does not track the many factors which are known to promote weight gain over time. Finally, there are potential selection biases in online recruitment, such as the reliance on the literacy and written communication abilities of participants which may mean that our findings are not representative of those autistic people who have difficulties with these media. It is also possible that our participants were individuals who have an interest in the topics of autism and weight, although the wide range of BMI scores and the preponderance of those in the Normal weight category suggest that this is not necessarily a key bias present in the sample.

In conclusion, our data are the first large-scale study which shows that autistic adults are more likely to be in all weight categories considered 'unhealthy', i.e., underweight, overweight, and obese, than they are to be in the healthy weight category. This is in line with a wealth of work which has examined the weight outcomes of autistic children and adolescents and extends our understanding of health across the lifespan for autistic people. In this study, there were no impacts on BMI from the interactions between selfreported mental health and autism status, suggesting that autistic people would likely benefit from the same interventions as non-autistic people without the assumption that they will inherently have mental health challenges. Future work should include qualitative explorations of the factors and experiences which autistic adults themselves feel contribute to their weight status to understand how to best support 
them regarding healthier choices, along with understanding whether autistic people want this kind of support at all.

Acknowledgements All authors would like to acknowledge the MRCMRF Fund (MR/R004595/1) and Swiss Anorexia (58/16) for making this research possible. In addition, KT would like to acknowledge Prof Tracey Wade and the Norman Munn Distinguished Visiting Scholar Award 2017/2018 from Flinders University South Australia. There are no conflicts of interest to declare for any author.

Funding All authors would like to acknowledge the MRC-MRF Fund (MR/R004595/1) and the Swiss Anorexia Foundation (58-16) for making this research possible. KT would like to acknowledge Prof Tracey Wade and the Norman Munn Distinguished Visiting Scholar Award 2017/2018 from Flinders University South Australia.

\section{Compliance with ethical standards}

Conflict of interest There are no conflicts of interest to declare.

Ethical approval All procedures involving human participants were in accordance with the ethical standards of the university and NHS Ethics Committee, and with the 1964 Helsinki Declaration and its later amendments.

Informed consent Informed consent was obtained from all participants over 16, parental consent and participant assent was obtained from all participants under 16 .

Open Access This article is distributed under the terms of the Creative Commons Attribution 4.0 International License (http://creativeco mmons.org/licenses/by/4.0/), which permits unrestricted use, distribution, and reproduction in any medium, provided you give appropriate credit to the original author(s) and the source, provide a link to the Creative Commons license, and indicate if changes were made.

\section{References}

1. Hill AP, Zuckerman KE, Fombonne E (2015) Obesity and autism. Pediatrics 136(6):1051-1061

2. Broder-Fingert S, Brazauskas K, Lindgren K, Iannuzzi D, Van Cleave J (2014) Prevalence of overweight and obesity in a large clinical sample of children with autism. Acad Pediatr 14(4):408-414

3. Curtin C, Jojic M, Bandini LG (2014) Obesity in children with autism spectrum disorder. Harv Rev Psychiatry 22(2):93-103

4. Cermak SA, Curtin C, Bandini LG (2010) Food selectivity and sensory sensitivity in children with autism spectrum disorders. J Am Diet Assoc 110(2):238-246

5. Johnson CR, Handen BL, Mayer-Costa M, Sacco K (2008) Eating habits and dietary status in young children with autism. J Dev Phys Disabil 20:437-448

6. Herndon AC, DiGuiseppi C, Johnson SL, Leiferman J, Reynolds A (2009) Does nutritional intake differ between children with autism spectrum disorders and children with typical development? J Autism Dev Disord 39(2):212-222

7. McCoy SM, Jakicic JM, Gibbs BB (2016) Comparison of obesity, physical activity, and sedentary behaviors between adolescents with autism spectrum disorders and without. J Autism Dev Disord 46(7):2317-2326
8. Petrina N, Carter M, Stephenson J (2014) The nature of friendship in children with autism spectrum disorders: a systematic review. Res Autism Spectr Disord 8(2):111-126

9. Eaves LC, Ho HH (2008) Young adult outcome of autism spectrum disorders. J Autism Dev Disord 38(4):739-747. https://doi. org/10.1007/s10803-007-0441-x

10. Croen LA, Zerbo O, Qian Y, Massolo ML, Rich S, Sidney S et al (2015) The health status of adults on the autism spectrum. Autism 19(7):814-823. https://doi.org/10.1177/1362361315577517

11. Howlin P, Moss P, Savage S, Rutter M (2013) Social outcomes in mid- to later adulthood among individuals diagnosed with autism and average nonverbal IQ as children. J Am Acad Child Adolesc Psychiatry 52(6):572-581.e1

12. Magiati I, Tay XW, Howlin P (2014) Cognitive, language, social and behavioural outcomes in adults with autism spectrum disorders: a systematic review of longitudinal follow-up studies in adulthood. Clin Psychol Rev 34(1):73-86

13. Howlin P (2000) Outcome in adult life for more able individuals with autism or asperger syndrome. Autism 4(1):63-83. https://doi. org/10.1177/1362361300004001005

14. Ells LJ, Lang R, Shield JPH, Wilkinson JR, Lidstone JSM, Coulton $\mathrm{S}$ et al (2006) Obesity and disability ? A short review. Obes Rev 7(4):341-345. https://doi.org/10.1111/j.1467789X.2006.00233.x

15. Piechota G, Małkiewicz J, Karwat ID (2005) Obesity as a cause and result of disability. Przegl Epidemiol 59(1):155-161

16. Allison DB, Newcomer JW, Dunn AL, Blumenthal JA, Fabricatore AN, Daumit GL et al (2009) Obesity among those with mental disorders: a national institute of mental health meeting report. Am J Prev Med 36(4):341-350

17. McElroy SL, Kotwal R, Malhotra S, Nelson EB, Keck PE, Nemeroff CB (2004) Are mood disorders and obesity related? A review for the mental health professional. J Clin Psychiatry 65(5):634-651

18. Kim JA, Szatmari P, Bryson SE, Streiner DL, Wilson FJ (2000) The prevalence of anxiety and mood problems among children with autism and asperger syndrome. Autism 4(2):117-132. https ://doi.org/10.1177/1362361300004002002

19. Gillott A, Standen PJ (2007) Levels of anxiety and sources of stress in adults with autism. J Intellect Disabil 11(4):359-370. https://doi.org/10.1177/1744629507083585

20. Ghaziuddin M, Zafar S (2008) Psychiatric comorbidity of adults with autism spectrum disorders. Vol. 5, Clinical Neuropsychiatry

21. Ghaziuddin M, Ghaziuddin N, Greden J (2002) Depression in persons with autism: implications for research and clinical care. $\mathrm{J}$ Autism Dev Disord 32(4):299-306

22. Gillberg C (1985) Autism and anorexia nervosa: related conditions? Nord Psykiatr Tidsskr 39(4):307-312

23. Oldershaw A, Treasure J, Hambrook D, Tchanturia K, Schmidt U (2011) Is anorexia nervosa a version of autism spectrum disorders? Eur Eat Disord Rev 19(6):462-474

24. Lang K, Stahl D, Espie J, Treasure J, Tchanturia K (2014) Set shifting in children and adolescents with anorexia nervosa: an exploratory systematic review and meta-analysis. Int J Eat Disord 47(4):394-399

25. Lopez C, Tchanturia K, Stahl D, Treasure J (2009) Weak central coherence in eating disorders: a step towards looking for an endophenotype of eating disorders. J Clin Exp Neuropsychol 31(1):117-125

26. Happé F, Frith U, Briskman J (2001) Exploring the cognitive phenotype of autism: weak "central coherence" in parents and siblings of children with autism: I. Experimental tests. J Child Psychol Psychiatry 42(3):S0021963001006916. https://doi. org/10.1017/S0021963001006916

27. Leppanen J, Sedgewick F, Treasure J, Tchanturia K (2018) Differences in the theory of mind profiles of patients with anorexia 
nervosa and individuals on the autism spectrum: a meta-analytic review. Neurosci Behav Rev 90:146-163

28. Westwood H, Lawrence V, Fleming C, Tchanturia K (2016) Exploration of friendship experiences, before and after illness onset in females with anorexia nervosa: a qualitative study. PLoS ONE 11(9):e0163528

29. Doris E, Westwood H, Mandy W, Tchanturia K (2014) A qualitative study of friendship in patients with anorexia nervosa and possible autism spectrum disorder. Psychology 5(11):1338

30. Andersen AE, Holman JE (1997) Males with eating disorders: challenges for treatment and research. Psychopharmacol Bull 33(3):391-397

31. Gould J, Ashton-Smith J (2018) Missed diagnosis or misdiagnosis? Girls and women on the autism spectrum. Good Autism Pract 31:34-41

32. Westwood H, Eisler I, Mandy W, Leppanen J, Treasure J, Tchanturia K (2016) Using the autism-spectrum quotient to measure autistic traits in anorexia nervosa: a systematic review and meta-analysis. J Autism Dev Disord 46(3):964-977. https://doi. org/10.1007/s10803-015-2641-0

33. Westwood H, Tchanturia K (2017) Autism spectrum disorder in anorexia nervosa: an updated literature review. Curr Psychiatry Rep 19(7):41. https://doi.org/10.1007/s11920-017-0791-9

34. American Psychiatric Association (2013) Diagnostic and statistical manual of mental disorders : DSM-5. American Psychiatric Association, Washington DC, p 947

35. Hoekstra RA, Vinkhuyzen AAE, Wheelwright S, Bartels M, Boomsma DI, Baron-Cohen S et al (2011) The construction and validation of an abridged version of the autism-spectrum quotient (AQ-Short). J Autism Dev Disord 41(5):589-596. https:// doi.org/10.1007/s10803-010-1073-0

36. Fairburn CG, Beglin SJ (1994) Assessment of eating disorders: interview or self-report questionnaire? Int J Eat Disord 16(4):363-370

37. Zigmond AS, Snaith RP (1983) The hospital anxiety and depression scale. Acta Psychiatr Scand 67(6):361-370

38. Team RC (2018) R: a language and environment for statistical computing, R Foundation for Statistical Computing, Austria, 2015. http://www.R-project.org

39. Ripley B, Venables B, Bates DM, Hornik K, Gebhardt A, Firth D, et al (2013) Package 'mass.' Cran R

40. Huber PJ, Ronchetti EM (1981) Robust statistics, ser. Wiley Ser Probab Math Stat New York, NY, USA Wiley-IEEE. 52:54

41. Tchanturia K, Adamson J, Leppanen J, Westwood H (2017) Characteristics of autism spectrum disorder in anorexia nervosa: a naturalistic study in an inpatient treatment programme. Autism 23:123-130. https://doi.org/10.1177/1362361317722431

42. Bentz M, Jepsen JRM, Pedersen T, Bulik CM, Pedersen L, Pagsberg AK et al (2017) Impairment of social function in young females with recent-onset anorexia nervosa and recovered individuals. J Adolesc Heal 60(1):23-32

43. Gillberg ICC, Gillberg C, Råstam M, Johansson M (1996) The cognitive profile of anorexia nervosa: a comparative study including a community-based sample. Compr Psychiatry 37(1):23-30

44. Westwood H, Stahl D, Mandy W, Tchanturia K (2016) The setshifting profiles of anorexia nervosa and autism spectrum disorder using the Wisconsin Card Sorting Test: a systematic review and meta-analysis. Psychol Med 46(9):1809-1827

45. Phillips KL, Schieve LA, Visser S, Boulet S, Sharma AJ, Kogan MD et al (2014) Prevalence and impact of unhealthy weight in a national sample of us adolescents with autism and other learning and behavioral disabilities. Matern Child Health J 18(8):19641975. https://doi.org/10.1007/s10995-014-1442-y

46. Spence C (2017) Comfort food: a review. Int J Gastron Food Sci 9:105-109

47. Locher JL, Yoels WC, Maurer D, van Ells J (2005) Comfort foods: an exploratory journey into the social and emotional significance of food. Food Foodways 13(4):273-297. https://doi. org/10.1080/07409710500334509

48. Leach LS, Christensen H, Mackinnon AJ, Windsor TD, Butterworth P (2008) Gender differences in depression and anxiety across the adult lifespan: the role of psychosocial mediators. Soc Psychiatry Psychiatr Epidemiol 43(12):983-998. https://doi. org/10.1007/s00127-008-0388-Z

49. George R, Stokes MA (2017) Gender identity and sexual orientation in autism spectrum disorder. Autism 22:970-982. https://doi. org/10.1177/1362361317714587

50. George R, Stokes M (2016) "Gender Is Not on My Agenda!": gender dysphoria and autism spectrum disorder. psychiatric symptoms and comorbidities in autism spectrum disorder. Springer, Champ, pp 139-150

51. Dewinter J, De Graaf H, Begeer S (2017) Sexual orientation, gender identity, and romantic relationships in adolescents and adults with autism spectrum disorder. J Autism Dev Disord 47(9):29272934. https://doi.org/10.1007/s10803-017-3199-9

52. Baldwin S, Costley D, Warren A (2014) Employment activities and experiences of adults with high-functioning autism and asperger's disorder. J Autism Dev Disord 44(10):2440-2449. https:// doi.org/10.1007/s10803-014-2112-z

53. Henninger NA, Taylor JL (2013) Outcomes in adults with autism spectrum disorders: a historical perspective. Autism 17(1):103116. https://doi.org/10.1177/1362361312441266

Publisher's Note Springer Nature remains neutral with regard to jurisdictional claims in published maps and institutional affiliations. 